\title{
Hawking radiation from the quantum Lemaître-Tolman-Bondi model
}

\author{
Claus Kiefer* and Jakob Müller-Hill† \\ Institut für Theoretische Physik, Universität zu Köln, \\ Zülpicher Str. 77, 50937 Köln, Germany. \\ T. P. Singh $\ddagger$ \\ Tata Institute of Fundamental Research, \\ Homi Bhabha Road, Mumbai 400 005, India. \\ Cenalo Var \\ RWC and Department of Physics, University of Cincinnati, \\ Cincinnati, Ohio 45221-0011, USA.
}

\begin{abstract}
In an earlier paper, we obtained exact solutions of the Wheeler-DeWitt equation for the Lemaître-Tolman-Bondi (LTB) model of gravitational collapse, employing a lattice regularization. In this paper, we derive Hawking radiation in non-marginally bound models from our exact solutions. We show that a non-vanishing energy function does not spoil the (approximate) Planck spectrum near the horizon. We can also reliably compute corrections to the Bogoliubov coefficient because our solutions are exact. The corrections are obtained by going beyond the near horizon region and are shown to introduce additional greybody factors, which modify the black body spectrum of radiation from the black hole.
\end{abstract}

PACS numbers: 04.60.Ds, 04.70.Dy

*Electronic address: kiefer@thp.uni-koeln.de

$\dagger$ Electronic address: jmh@thp.uni-koeln.de

${ }^{\ddagger}$ Electronic address: tpsingh@nagaum.tifr.res.in

${ }^{\S}$ Electronic address: vaz@physics.uc.edu 


\section{INTRODUCTION}

A consistent quantum theory of gravity should provide us with a clear picture of blackhole evaporation and black-hole entropy. The main approaches to quantum gravity have up to now only led to partial successes [1], concerning mainly the statistical explanation for the entropy from microscopic degrees of freedom.

In view of the fact that a generally agreed upon quantum theory of gravity does not yet exist, it is important to examine the quantization of particular models of physical interest by applying established quantization techniques. Among the most interesting models are those with spherical symmetry because they are sufficiently non-trivial and, at the same time, are simple enough to be tractable. One such model is that of Lemaître, Tolman and Bondi (LTB), which describes a self-gravitating cloud of non-interacting dust. It was first introduced by Lemaître [2] in an attempt to describe cosmology, where it has found interesting applications [3]. It also describes gravitational collapse, but a full understanding of this process requires the application of quantum gravity. Given our limited understanding of quantum gravity at present, perhaps the approach that is most suited for addressing quantum collapse and questions concerning its final fate is canonical quantum general relativity. Although limited in its scope as a theory of quantum gravity, the canonical theory can meaningfully address some open questions regarding singularities in the quantum theory, such as the possibility of singularity avoidance, the quantum evolution of black holes, and the role of naked singularities in quantum gravity.

In a recent paper we presented an exact canonical quantization of the LTB model [4]. Earlier work [5, 6, 7, 8] had shown that the classical geometrodynamic constraints of this dust system, the Hamiltonian constraint and the momentum constraint, are both given in terms of a canonical chart consisting of the mass contained within spherical shells, the physical shell radius, the dust proper time and their conjugate momenta. The momentum conjugate to the mass function may be eliminated in the Hamiltonian constraint using the momentum constraint, and this procedure was shown to lead to a new and simpler constraint which is able to take the place of the original Hamiltonian constraint. Dirac's constraint quantization then yields the Wheeler-DeWitt equation for the wave functional describing the quantum collapse.

In [4] this approach was sharpened and particular attention was paid to the regularization of the Wheeler-DeWitt equation and diffeomorphism invariance. Regularization was

performed on a one-dimensional lattice following a suggestion in [9], and special care was taken to ensure that the momentum constraint is fulfilled in the continuum limit. As a consequence, the lattice wave functional becomes described in terms of three equations, the Hamilton-Jacobi equation and two additional constraints which make it possible to obtain exact solutions for a particular factor ordering. In this paper we use these exact solutions to examine Hawking evaporation during the collapse. The evaporation problem was also addressed by us in [10], but only for marginally bound collapse in the WKB approximation. Here we consider the generic case, that is, the case including the non-marginal models for which the classical shells start with a non-vanishing velocity at infinity.

Our paper is organized as follows. In Sec. II we review the major features of the quantum 
LTB model. We present, in particular, the exact quantum states found in [4]. Sec. III is the main part of our paper. We show in detail how the spectrum of Hawking radiation together with appropriate greybody factors can be retrieved from these exact quantum states. This provides a bridge from quantum gravity (represented here by the LTB model) to semiclassical gravity and the Hawking effect. Sec. IV gives a summary of the obtained results and an outlook on potential future developments.

\section{THE CLASSICAL AND QUANTUM LTB MODEL}

\section{A. The classical LTB model}

The LTB model describes a self-gravitating dust cloud. Its energy-momentum tensor reads $T_{\mu \nu}=\epsilon(\tau, \rho) u_{\mu} u_{\nu}$, where $u^{\mu}=u^{\mu}(\tau, \rho)$ is the four-velocity vector of a dust particle with proper time $\tau$ and labeled by $\rho$ ( $\rho$ thus labels the various shells that together form the dust cloud). The line element for the LTB spacetime is given by

$$
d s^{2}=-d \tau^{2}+\frac{\left(\partial_{\rho} R\right)^{2}}{1+2 E(\rho)} d \rho^{2}+R^{2}(\rho)\left(d \theta^{2}+\sin ^{2} \theta d \phi^{2}\right)
$$

and the Einstein equations lead to

$$
8 \pi G \epsilon(\tau, \rho)=\frac{\partial_{\rho} F}{R^{2} \partial_{\rho} R} \quad \text { and } \quad\left(\partial_{\tau} R\right)^{2}=\frac{F}{R}+2 E,
$$

where $F(\rho) \equiv 2 G M(\rho)$ is some non-negative function. We set $c=1$ throughout. The case of collapse is described by $\partial_{\tau} R(\tau, \rho)<0$.

There exists still the freedom to rescale the shell index $\rho$. This freedom can be fixed by demanding

$$
R(0, \rho)=\rho,
$$

so that for $\tau=0$ the label coordinate $\rho$ is equal to the curvature radius $R$. Now we can express the functions $F(\rho)$ and $E(\rho)$ in terms of the energy density $\epsilon$ at $\tau=0$. From (2) one gets

$$
\begin{aligned}
& F(\rho)=8 \pi G \int_{0}^{\rho} \epsilon(0, \tilde{\rho}) \tilde{\rho}^{2} d \tilde{\rho}, \\
& E(\rho)=\left[\partial_{\tau} R(\tau=0, \rho)\right]^{2}-\frac{8 \pi G}{\rho} \int_{0}^{\rho} \epsilon(0, \tilde{\rho}) \tilde{\rho}^{2} d \tilde{\rho} .
\end{aligned}
$$

The interpretation of these quantities is that $F(\rho) / 2 G$ is the active gravitating mass inside of $R(\tau, \rho)$, while $E(\rho)$ is the total energy per unit mass of the shell labeled by $\rho$. The marginally bound models are defined by $E(\rho)=0$. In the present paper we discuss the general case which includes the non-marginal case defined by $E(\rho) \neq 0$.

The solution of (2) is given in [4] and examined in great detail in [11]. The end state of collapse is either a black hole or a naked singularity, depending on the initial parameters governing the collapse. At a finite dust proper time $\tau=\tau_{0}(\rho)$, the shell labeled by $\rho$ reaches 
a curvature radius $R=0$, which results in a curvature singularity. Therefore $\tau$ can only take values between $-\infty$ and $\tau_{0}(\rho)$.

The canonical formalism begins with the general ansatz for a spherically-symmetric line element,

$$
d s^{2}=-N^{2} d t^{2}+L^{2}\left(d r-N^{r} d t\right)^{2}+R^{2} d \Omega^{2},
$$

where $N$ and $N^{r}$ are the lapse and shift function, respectively. The total action is the Einstein-Hilbert action together with an action describing the dust. The phase space consists of the three functions $L(t, r)$ and $R(t, r)$ in ([6) , the dust proper time, $\tau(t, r)$, and their canonical momenta $P_{L}, P_{R}$, and $P_{\tau}$. In the following, a prime will denote a derivative with respect to $r$. All variables are functions of $t$ and $r$. After a series of canonical transformations, performed in the spirit of Kuchař's reduction of static spherical geometries [5], it becomes possible to describe the phase space by a different chart, consisting of the dust proper time, $\tau(r)$, the physical radius, $R(r)$, the mass density function, $\Gamma=F^{\prime}(r)$ and their canonical momenta. The collapse problem is reduced to two classical constraints,

$$
\begin{aligned}
H & =\left(P_{\tau}^{2}+\mathcal{F} \bar{P}_{R}^{2}\right)-\frac{\Gamma^{2}}{4 \mathcal{F}} \approx 0, \\
H_{r} & =\tau^{\prime} P_{\tau}+R^{\prime} \bar{P}_{R}-\Gamma P_{\Gamma}^{\prime} \approx 0,
\end{aligned}
$$

where $\mathcal{F}=1-F / R, \bar{P}_{R}$ is a new canonical momentum for $R$, which follows via a canonical transformation from the original variables, and $P_{\Gamma}$ is the momentum conjugate to $\Gamma$. This transformation also absorbs a boundary term which is present in the original chart [4].

\section{B. Relation between dust proper time and Killing time}

Under some circumstances, the momentum $P_{\Gamma}$ has the interpretation of a Killing time. In this subsection we explain under which circumstances this interpretation makes sense. In the new variables, the proper time is constrained by the expression

$$
\tau^{\prime}=2 P_{\Gamma}^{\prime} \sqrt{1+2 E} \pm \frac{R^{\prime}}{\mathcal{F}} \sqrt{1+2 E-\mathcal{F}} .
$$

Defining $a \equiv 1 / \sqrt{1+2 E}$ gives

$$
\tau^{\prime}=\frac{2 P_{\Gamma}^{\prime}}{a} \pm R^{\prime} \frac{\sqrt{1-a^{2} \mathcal{F}}}{a \mathcal{F}} .
$$

If the mass density vanishes for all $r$ greater than a given $r_{b}$, and if $E$ is constant for all $r$ greater than $r_{b}$, Eq. (10) can be integrated. This yields

$$
\begin{aligned}
& a \tau=2 P_{\Gamma} \pm \int d R \frac{\sqrt{1-a^{2} \mathcal{F}}}{\mathcal{F}} \\
&=2 P_{\Gamma} \pm F\left(\frac{\sqrt{1-a^{2} \mathcal{F}}}{1-\mathcal{F}}+\ln \left|\frac{1-\sqrt{1-a^{2} \mathcal{F}}}{1+\sqrt{1-a^{2} \mathcal{F}}}\right|\right. \\
&\left.\quad-\frac{1-a^{2} / 2}{\sqrt{1-a^{2}}} \ln \left|\frac{\sqrt{1-a^{2} \mathcal{F}}-\sqrt{1-a^{2}}}{\sqrt{1-a^{2} \mathcal{F}}+\sqrt{1-a^{2}}}\right|\right),
\end{aligned}
$$


where the upper sign refers to infalling dust and the lower sign to outgoing dust. Here we have assumed that $0<a \leq 1$ (corresponding to $E \geq 0$ ). The Einstein equations guarantee that the discriminant $1-a^{2} \mathcal{F}$ is non-negative. (A similar analysis can be carried out for $-1 / 2<E<0[4])$.

We know from Birkhoff's theorem that the spacetime around a collapsing dust cloud is given by the Schwarzschild solution. In [5] it was shown for the Schwarzschild geometry that $2 P_{\Gamma}$ is equal to the Killing time $T$. Thus (111) connects the dust proper time $\tau$ with the Killing time $2 P_{\tau}$ at the boundary $r_{b}$. For small $\Gamma$ and $E^{\prime}$ the relationship may still be used, since then we have a small amount of dust propagating in a Schwarzschild background; only in this case the concept of Killing time makes sense. This is the situation to be discussed in Sec. III. In the limit $a \rightarrow 1(E \rightarrow 0)$ we obtain

$$
\tau=T+2 \sqrt{F}\left[\sqrt{R}-\frac{\sqrt{F}}{2} \ln \left(\frac{\sqrt{R}+\sqrt{F}}{\sqrt{R}-\sqrt{F}}\right)\right]
$$

which is identical to the relation used in [10] for the marginal case. The plus sign after the $T$ on the right-hand side has been chosen in order to describe a collapsing dust cloud.

The transformation (11) can also be interpreted in the following way. For a Schwarzschild spacetime it gives a relation between Killing time and the time used by families of freely falling observers. Each family is characterized by a fixed value of $E$. All observers within one family start at infinity with the speed $v_{\infty}$, where $E=v_{\infty}^{2} /\left(2\left(1-v_{\infty}^{2}\right)\right)$. In the marginal case they thus start with zero initial speed. In this case (13) gives the relation between the Schwarzschild time and the Painlevé-Gullstrand time [13].

\section{The quantum LTB model}

Dirac's quantization procedure may be employed to turn the classical constraints in (7) and (8) into quantum constraints which act on wave functionals. The Hamiltonian constraint (7) then leads to the Wheeler-DeWitt equation, and the momentum constraint (8) leads to a quantum equation which imposes diffeomorphism invariance on the wave functional.

The resulting quantum constraint equations are of a purely formal nature. They must therefore be regularized before solutions can be obtained. Following a suggestion in [9] we have considered in [4] a one-dimensional lattice given by a discrete set of points $r_{i}$ separated by a distance $\sigma$. In order that the momentum constraint is fulfilled in the continuum limit, it is important to start with a corresponding ansatz for the wave functional before putting it on the lattice. We therefore made the ansatz

$$
\Psi[\tau(r), R(r), \Gamma(r)]=U\left(\int d r \Gamma(r) \mathcal{W}(\tau(r), R(r), \Gamma(r))\right)
$$

where $U: \mathbb{R} \rightarrow \mathbb{C}$ is at this stage some arbitrary (differentiable) function. The ansatz has to be compatible with the lattice, which means that it has to factorize into different functions 
for each lattice point. So we have to make the choice $U=\exp$, which gives

$$
\begin{aligned}
& \Psi[\tau(r), R(r), \Gamma(r)] \\
& =\lim _{\sigma \rightarrow 0} \prod_{i} \exp \left(\sigma \Gamma_{i} \mathcal{W}_{i}\left(\tau\left(r_{i}\right), R\left(r_{i}\right), F\left(r_{i}\right)\right)\right) \\
& =\lim _{\sigma \rightarrow 0} \prod_{i} \Psi_{i}\left(\tau\left(r_{i}\right), R\left(r_{i}\right), \Gamma\left(r_{i}\right), F\left(r_{i}\right)\right)
\end{aligned}
$$

where

$$
F\left(r_{i}\right)=\sum_{j=0}^{i} \sigma \Gamma_{j}
$$

The lattice version of the Wheeler-DeWitt equation was then found in [4] to read

$$
\left[G \hbar^{2}\left(\frac{\partial^{2}}{\partial \tau_{j}^{2}}+\mathcal{F}_{j} \frac{\partial^{2}}{\partial R_{j}^{2}}+A\left(R_{j}, F_{j}\right) \frac{\partial}{\partial R_{j}}\right)+B(R, F)+\frac{\sigma^{2} \Gamma}{4 G \mathcal{F}_{j}}\right] \Psi_{j}=0
$$

where $\mathcal{F}_{j}=1-F_{j} / R_{j}>0$ (outside the horizon). Making the ansatz (17) together with the redefinition $\mathcal{W}=i W / 2$, we found in [4] that in order for the resulting equation to be independent of the choice of $\sigma$ (and thus also in the limit $\sigma \rightarrow 0$ ) a set of three equations must be satisfied. If one finds solutions to all three equations, one can do all other calculations on the lattice, since these solutions have a well defined continuum limit and satisfy the momentum constraint. In [4] such solutions were found for a particular factor ordering in the Wheeler-DeWitt equation. (The solutions found in [9] do not fulfill the momentum constraints in the continuum limit.)

The general solutions within the ansatz (14), with $U=$ exp and satisfying the constraints, were found to be of the form

$$
\begin{aligned}
\Psi[\tau, R, \Gamma]= & \exp \left(\int d r b(F(r)) \Gamma / G \hbar\right) \\
& \times \exp \left\{\mp \frac{i}{2 G \hbar} \int d r \Gamma\left[a(F(r)) \tau \pm \int^{R} d R \frac{\sqrt{1-a^{2}(F(r)) \mathcal{F}}}{\mathcal{F}}\right]\right\}
\end{aligned}
$$

where we have introduced an implicit dependence $a(F(r))$ in order to get solutions fulfilling the momentum constraint, and $b(F(r))$ is an arbitrary function. They serve as the starting point for the discussion of the Hawking radiation in the next section.

\section{DERIVATION OF HAWKING RADIATION FOR THE GENERAL LTB MODEL}

\section{A. Preliminaries}

The exact quantum states (20) describe a generic situation. In order to study Hawking radiation in this framework, we need to introduce into the formalism the concept of a black hole and the analogue of the quantum fields used in the standard treatment. Following [10], 
we consider a Schwarzschild black hole plus some dust perturbation. The dust perturbation mimics the quantum fields in Hawking's derivation [14]. Formally, this perturbation is described by the variable $\Gamma \equiv F^{\prime}$ in which the constant mass of the black hole drops out. In analogy to [10], the black-hole state factors out in (20), and the remaining state will describe Hawking radiation. This remaining state then assumes the form of (20) in which $F$ can approximately be replaced by $2 G$ times the black-hole mass.

The central idea in [14] was the use of a Bogoliubov transformation of field operators in the Schwarzschild spacetime. Here, the situation is different, since we have full quantum gravitational states at our disposal. We thus have to first identify those quantum states that correspond to the ingoing and outgoing modes, respectively, of the standard approach. Then we have to calculate their inner product. Since the description should refer to observers at infinity, the inner product will be evaluated on hypersurfaces of constant Killing (Schwarzschild) time $T$, and not on hypersurfaces of constant dust time $\tau$ (which corresponds to freely falling observers).

We would therefore like to re-express our solutions in terms of $T$. For the expanding cloud we have the connection between dust time $\tau$ and Killing time $T$ given in (12):

$$
a \tau=T-\int^{R} d R \frac{\sqrt{1-a^{2} \mathcal{F}}}{\mathcal{F}} .
$$

The relevant exact solutions of the Wheeler-DeWitt equation read

$$
\Psi^{ \pm}=\exp \left(\frac{1}{G \hbar} \int d r b \Gamma \mp \frac{i}{2 G \hbar} \int d r \Gamma\left[a \tau \pm \int^{R} d R \frac{\sqrt{1-a^{2} \mathcal{F}}}{\mathcal{F}}\right]\right)
$$

In principle one has to include additional normalization factors $N^{+}$and $N^{-}$for the wave functionals. Since it is not clear how to normalize the states properly in the full theory, we leave this point open here; it will not be crucial for our results derived below.

In order to be as close as possible to the standard derivation of Hawking radiation, we want to obtain the scattered outgoing part of the 'ingoing wave functional'. We choose $\Psi^{+}$from (22) as in-going wave functional of positive frequency. This is justified as follows. Inserting the relation (21) into the corresponding expression in (22), we obtain

$$
\Psi^{+}=\exp \left(\frac{1}{G \hbar} \int d r b \Gamma-\frac{i}{2 G \hbar} \int d r \Gamma T\right)
$$

which has the standard form of a positive-frequency wave function. Note that this wave functional is independent of $R$. The lattice version of this state is obtained through the replacement of $\Gamma=F^{\prime}$ by a dimensionless quantity, $\omega$. This leads to

$$
\Psi_{\sigma \omega}^{+}=\lim _{\sigma \rightarrow 0} \prod_{i} e^{-2 b_{i} \sigma \omega_{i} F_{i} / G \hbar-i \sigma \omega_{i} T_{i} / G \hbar}
$$

see (17). Our result will be a product of the results for each of the shells which together form the dust cloud. 
For the out-going modes of negative frequency we have to take the state $\Psi^{-}$. Inserting (21) into the corresponding state of (22) then gives

$$
\Psi^{-}=\exp \left(\frac{1}{G \hbar} \int d r b \Gamma+\frac{i}{2 G \hbar} \int d r \Gamma\left[T-2 \int^{R} d R \frac{\sqrt{1-a^{2} \mathcal{F}}}{\mathcal{F}}\right]\right) .
$$

The corresponding lattice version reads

$$
\Psi_{\sigma \omega}^{-}=\lim _{\sigma \rightarrow 0} \prod_{i} \exp \left(-\frac{2 b_{i} \sigma \omega_{i} F_{i}}{G \hbar}-\frac{i \sigma \omega_{i}}{G \hbar}\left[T_{i}-2 \int^{R} d R_{i} \frac{\sqrt{1-a_{i}^{2} \mathcal{F}_{i}}}{\mathcal{F}_{i}}\right]\right) .
$$

In Sec. III.B we shall calculate the overlap of in- and out-going states in the near-horizon limit, while in Sec. III.C we give a general expression for the result away from the horizon.

\section{B. The Bogoliubov coefficient in the near-horizon limit.}

We shall now consider the analogue of the usual Bogolubov coefficient $\beta_{\omega \omega^{\prime}}$ on each shell. We define it as the inner product between the in-going wave functional with positive frequency and the out-going wave functional with negative frequency (both frequencies are present in the Wheeler-DeWitt equation because of the $P_{\tau}^{2}$-term there). In the standard

approach one normalizes the field modes according to the Klein-Gordon inner product [15]. For solutions of the Klein-Gordon equation in $1+1$ dimensions,

$$
\phi_{k}(x, t)=u_{k}(x) e^{-i \omega_{k} t},
$$

the spatial modes are normalized according to

$$
\left\langle u_{k}, u_{k^{\prime}}\right\rangle=\frac{\delta\left(k-k^{\prime}\right)}{2 \omega_{k}},
$$

from which one gets for the Bogoliubov coefficient

$$
\beta_{k k^{\prime}}=2 \omega_{k}\left\langle u_{k}^{*}, \bar{u}_{k^{\prime}}\right\rangle,
$$

where $\bar{u}_{k^{\prime}}$ denotes the 'new' set of field modes with respect to which the solution of the Klein-Gordon equation is expanded. We thus define in our case

$$
\beta_{\omega \omega^{\prime}}=\frac{2 \sigma \omega}{G \hbar} \int_{F}^{\infty} d R \sqrt{g_{R R}} \Psi_{\sigma \omega}^{-*} \Psi_{\sigma \omega^{\prime}}^{+}
$$

Since we shall evaluate $\beta$ separately for each shell, we skip the shell index $i$ here and in the following. Note that $\sigma \omega / G \hbar$ replaces the $\omega$ of the standard approach; it possesses the correct dimension of an inverse length, so that $\beta$ itself is dimensionless, as it must be. The integration goes from the horizon $(\mathcal{F}=0)$ to infinity.

In order to specify the measure of integration, we recall again that the inner product should be evaluated on a $T=$ constant hypersurface [10]. Performing the coordinate transformation $(R, \tau) \mapsto(R, T)$, we get with the help of (21) the result

$$
\sqrt{g_{R R}}=\sqrt{1+2 E} \frac{R}{R-F} .
$$


We are now ready to evaluate the integral (27). Inserting the wave functionals in (24) and (26) into (27) then gives

$$
\begin{aligned}
& \beta_{\omega \omega^{\prime}}=2 \omega \sqrt{1+2 E} \exp \left(-\frac{2 b \sigma F\left(\omega+\omega^{\prime}\right)}{G \hbar}-i \frac{\sigma T\left(\omega+\omega^{\prime}\right)}{G \hbar}\right) \times \\
& \int_{F}^{\infty} d R \frac{R}{R-F} \exp \left[\frac{2 i \sigma \omega}{G \hbar} \int^{R} d R \frac{\sqrt{1-a^{2} \mathcal{F}}}{\mathcal{F}}\right] .
\end{aligned}
$$

This is the exact expression, but it is too complicated to compute in the given form. However, in order to investigate black-hole radiation, a near-horizon approximation is sufficient [10]. Anyway, we shall derive in the next subsection an exact, albeit less tractable, result. In order to evaluate $\beta$, we introduce the variable

$$
s=\sqrt{\frac{R}{F}}-1
$$

which ranges from zero (horizon) to infinity. The Bogoliubov coefficient, written in terms of $s$, then becomes

$$
\begin{aligned}
& \beta_{\omega \omega^{\prime}}=2 \omega \sqrt{1+2 E} \exp \left(-\frac{2 b \sigma F\left(\omega+\omega^{\prime}\right)}{G \hbar}-i \frac{\sigma T\left(\omega+\omega^{\prime}\right)}{G \hbar}\right) \times \\
& 2 F \int_{0}^{\infty} d s \frac{(1+s)^{3}}{s^{2}+2 s} \exp \left[\frac{4 i \sigma \omega F}{G \hbar}\left(\int^{s} d \tilde{s}(1+\tilde{s})^{2} \frac{\sqrt{(1+\tilde{s})^{2}-a^{2}\left[(1+\tilde{s})^{2}-1\right]}}{\tilde{s}^{2}+2 \tilde{s}}\right)\right] .
\end{aligned}
$$

We have not specified the lower limit in the $\tilde{s}$-integral, since it will only contribute a phase to $\beta$, which will not contribute to its absolute square.

The near-horizon approximation is then described by an expansion with respect to $s$. This gives

$$
\begin{aligned}
\beta_{\omega \omega^{\prime}}= & \frac{2 F \sigma \omega}{G \hbar} \sqrt{1+2 E} \exp \left(-\frac{2 b \sigma F\left(\omega+\omega^{\prime}\right)}{G \hbar}-i \frac{\sigma T\left(\omega+\omega^{\prime}\right)}{G \hbar}\right) \times \\
& \int_{0}^{\infty} d s s^{-1+2 i \sigma \omega F / G \hbar} \exp \left[i \frac{\sigma \omega F}{G \hbar} \frac{3+10 E}{1+2 E} s\right] .
\end{aligned}
$$

In order to evaluate the integral in (31), we insert a regularization factor $\exp (-p s), p>0$, which guarantees convergence at the upper limit. We use the formula [16]

$$
\int_{0}^{\infty} d x x^{\nu-1} e^{-(p+i q) x}=\Gamma(\nu)\left(p^{2}+q^{2}\right)^{-\nu / 2} e^{-i \nu \arctan (q / p)}
$$

and perform after integration the limit $p \rightarrow 0$. We then arrive at the following expression:

$$
\begin{aligned}
& \beta_{\omega \omega^{\prime}}=\frac{2 F \sigma \omega}{G \hbar} \sqrt{1+2 E} \exp \left(-\frac{2 b \sigma F\left(\omega+\omega^{\prime}\right)}{G \hbar}-i \frac{\sigma T\left(\omega+\omega^{\prime}\right)}{G \hbar}\right) \times \\
& \Gamma\left(\frac{2 i \sigma \omega F}{G \hbar}\right)\left(\frac{\sigma \omega F}{G \hbar} \frac{3+10 E}{1+2 E}\right)^{-2 i \sigma \omega F / G \hbar} e^{-\pi \sigma \omega F / G \hbar} .
\end{aligned}
$$


For the absolute square of $\beta$ we then get

$$
\left|\beta_{\omega \omega^{\prime}}\right|^{2}=\frac{4 \pi F \sigma \omega(1+2 E)}{G \hbar} \frac{e^{-4 b \sigma F\left(\omega+\omega^{\prime}\right) / G \hbar}}{e^{4 \pi \sigma \omega F / G \hbar}-1} .
$$

In order to calculate the particle-creation rate, we have to evaluate the expression $\sum_{k} \beta_{i k} \beta_{i k}^{*}$, which here corresponds to the integral $\int_{0}^{\infty} d \omega^{\prime}\left|\beta_{\omega \omega^{\prime}}\right|^{2}$ (without further factors, in order to keep the result dimensionless). We get

$$
\left\langle\text { in }\left|\hat{N}_{\text {out }}\right| \text { in }\right\rangle=\frac{\pi \omega(1+2 E)}{b} \frac{e^{-4 b \sigma F \omega / G \hbar}}{e^{4 \pi \sigma \omega F / G \hbar}-1} .
$$

where $\hat{N}_{\text {out }}$ denotes the 'out'-particle-number operator.

We recognize immediately that the spectrum contains a Planckian (thermal) factor and that the associated temperature is independent of $E$, as expected. In fact, the only $E$ dependent term is in the prefactor.

Replacing $\sigma \omega$ by $G \Delta \epsilon$, where $\Delta \epsilon$ is the energy of a shell, we arrive at the final result

$$
\left\langle\text { in }\left|\hat{N}_{\text {out }}\right| \text { in }\right\rangle=\frac{\pi G \Delta \epsilon(1+2 E)}{\sigma b} \frac{e^{-8 b G M \Delta \epsilon / \hbar}}{e^{8 \pi G M \Delta \epsilon / \hbar}-1},
$$

where we have set $F=2 G M$. From here we read off the standard Hawking temperature for a Schwarzschild black hole:

$$
k_{\mathrm{B}} T_{\mathrm{H}}=\frac{\hbar}{8 \pi G M},
$$

which holds for each shell separately.

We recognize that the Planckian spectrum is modified by greybody factors which explicitly depend on $\Delta \epsilon$. Greybody factors occur in the standard treatment by taking into account the back-scattering of field modes into the black hole; see, for example, [17]. Our greybody factors are different in origin compared to those coming from back-scattering, since we derived them from solutions of the Wheeler-DeWitt equation for gravity with quantum dust.

At this stage, the number $b$ has not been fixed further; it occurs in the full quantum gravitational state and depends on the imposed boundary conditions. The simplest choice for $b$ is $b=0$. In this case the integral over $\omega^{\prime}$ diverges. Dividing out the infinite constant arising from this integration, one arrives for $b=0$ at the result

$$
\left\langle\text { in }\left|\hat{N}_{\text {out }}\right| \text { in }\right\rangle=\frac{8 \pi G M \Delta \epsilon(1+2 E)}{\hbar} \frac{1}{e^{8 \pi G M \Delta \epsilon / \hbar}-1} .
$$

This corresponds to the result obtained in the WKB approximation, see [10] (after taking into account that the $\beta$ there differs from the $\beta$ here by a factor of $2 \omega$ ).

\section{Exact expression for the Bogoliubov coefficient}

It is not difficult to obtain the exact expression for the Bogoliubov coefficient by considering the series expansion in $s$ about $s=0$ for arbitrary $E$ of the integrand in (30). The 
measure can be expanded as

$$
\frac{2(1+s)^{3}}{s^{2}+2 s}=\frac{1}{s}+\frac{5}{2}+\frac{7 s}{4}+\ldots \stackrel{\text { def }}{=} \sum_{n=-1}^{\infty} \alpha_{n} s^{n}
$$

while the exponent becomes

$$
\begin{aligned}
& \frac{4 i \sigma \omega F}{G \hbar}\left(\frac{1}{2} \ln s+\frac{1}{4}\left(5-2 a^{2}\right) s+\frac{1}{16}\left(7-4 a^{2}-2 a^{4}\right) s^{2}+\ldots\right) \\
\stackrel{\text { def }}{=} & \frac{2 i \sigma \omega F}{G \hbar}\left(\ln s+\sum_{m=1}^{\infty} \beta_{m} s^{m}\right),
\end{aligned}
$$

where we have returned to our notation $a=1 / \sqrt{1+2 E}$. From (30) we see that we must perform the integration

$$
F \sum_{n=-1}^{\infty} \alpha_{n} \int_{0}^{\infty} d s s^{n+2 i \sigma \omega F / G \hbar} e^{2 i \sigma \omega F \beta_{1} s / G \hbar} e^{2 i \sigma \omega F \sum_{m=2}^{\infty} \beta_{m} s^{m} / G \hbar}
$$

where $\beta_{1}=\frac{1}{2}\left(5-2 a^{2}\right)=\frac{1}{2}(3+10 E) /(1+2 E)$. This is best done by letting

$$
e^{2 i \sigma \omega F \sum_{m=2}^{\infty} \beta_{m} s^{m} / G \hbar}=1+\frac{2 i \sigma \omega F}{G \hbar} \sum_{m=2}^{\infty} \beta_{m} s^{m}+\frac{1}{2}\left(\frac{2 i \sigma \omega F}{G \hbar}\right)^{2} \sum_{m, n=2}^{\infty} \beta_{m} \beta_{n} s^{m} s^{n} \equiv \sum_{m=0}^{\infty} \gamma_{m} s^{m},
$$

where all the coefficients $\gamma_{m}$ can be evaluated in principle from the coefficients $\beta_{m}$, and $\gamma_{0}=1, \gamma_{1}=0$. We then find the integral

$$
\begin{aligned}
& =F \sum_{n, m=0}^{\infty} \alpha_{n-1} \gamma_{m} \int_{0}^{\infty} d s s^{-1+n+m+2 i \sigma \omega F / G \hbar} e^{2 i \sigma \omega F \beta_{1} s / G \hbar} \\
& =\left.F \sum_{n, m=0}^{\infty} \alpha_{n-1} \gamma_{m}\left(-i \frac{\partial}{\partial \chi}\right)^{n+m} \int_{0}^{\infty} d s s^{-1+2 i \sigma \omega F / G \hbar} e^{i \chi s}\right|_{\chi=2 \sigma \omega F \beta_{1} / G \hbar} .
\end{aligned}
$$

In this form, it can be evaluated as before. The first term $(n=0=m)$ is the near horizon approximation given earlier. The exact Bogoliubov coefficient can be written as the (infinite) sum

$$
\begin{aligned}
\beta_{\omega \omega^{\prime}}= & \frac{2 F \sigma \omega}{G \hbar} \sqrt{1+2 E} \exp \left(-\frac{2 b \sigma F\left(\omega+\omega^{\prime}\right)}{G \hbar}-i \frac{\sigma T\left(\omega+\omega^{\prime}\right)}{G \hbar}\right) \times \\
& \left(\frac{2 \sigma \omega F \beta_{1}}{G \hbar}\right)^{-2 i \sigma \omega F / G \hbar} e^{-\pi \sigma \omega F / G \hbar} \Gamma\left(\frac{2 i \sigma \omega F}{G \hbar}\right) \times \\
& {\left[1+\sum_{n+m=1}^{\infty}(-i)^{n+m} \alpha_{n-1} \gamma_{m}\left(-\frac{2 i \sigma \omega F}{G \hbar}\right) \ldots\left(-\frac{2 i \sigma \omega F}{G \hbar}-n-m+1\right)\left(\frac{2 \sigma \omega F \beta_{1}}{G \hbar}\right)^{-n-m}\right] . }
\end{aligned}
$$


The simplest correction term to the near-horizon approximation is obtained for $n=1$, $m=0$ :

$$
(-i) \alpha_{0} \gamma_{0}\left(-\frac{2 i \sigma \omega F}{G \hbar}\right)\left(\frac{2 \sigma \omega F \beta_{1}}{G \hbar}\right)^{-1}=-\frac{5}{2 \beta_{1}} .
$$

Since $1-5 / 2 \beta_{1}=-2 /(3+10 E)$, this leads to a $\omega$-independent correction term in $|\beta|^{2}$ given by

$$
\frac{4}{(3+10 E)^{2}} \leq \frac{4}{9}
$$

In general, the ensuing correction terms in (43) depend on $\omega$ (and thus on the shell energy $\Delta \epsilon)$ and therefore lead to a non-trivial modification of the greybody factors. In the limit of $\omega \rightarrow \infty$, the correction terms are independent of $\omega$.

Such a kind of greybody factors cannot be found in the standard derivation of Hawking radiation [14], since there the geometric-optics approximation is strictly assumed. We can perform such a calculation because we have an exact quantum state at our disposal.

We finally mention that no 'trans-Planckian problem' occurs here, since the whole calculation is based on genuine quantum gravitational states, and thus all Planck-scale effects are automatically included, at least within the limits given by our model.

\section{SUMMARY AND OUTLOOK}

The programme for the canonical quantization of the LTB model was started in [8], following methods earlier developed in [5]. The classical Hamiltonian constraint and momentum constraint were given in terms of a canonical chart consisting of the mass, radius, dust proper time, and their conjugate momenta. The momentum conjugate to the mass function was eliminated from the Hamiltonian constraint using the momentum constraint, and this procedure led to a new and simpler Hamiltonian constraint. The Wheeler-DeWitt equation for the wave functional describing quantum dust collapse then follows from the Dirac constraint quantization of the Hamiltonian constraint.

To make progress towards finding solutions to the Wheeler-DeWitt equation, one must adopt a regularization scheme. The simplest choice is the delta-function regularization, which was used in [8], but which turns out to be equivalent to the WKB approximation. The solutions for the marginal case in this approximation were obtained in [8] and were used to derive Hawking radiation in [10]. In order to arrive at genuine quantum gravity effects one clearly needs to go beyond the WKB approximation.

With this intent, a lattice regularization was proposed in [9]. A class of exact solutions to the Wheeler-DeWitt equation for the general non-marginal case were worked out in [4]. In order to construct such solutions, one assumes that the wave functional of the collapsing dust cloud can be written as a product of the wave functions of individual shells. We then look for solutions which are separable in $\tau$ and $R$; it turns out that for the momentum constraint to be satisfied in the continuum limit, a specific factor-ordering must be chosen, which happens to depend on the energy function $E$. Interestingly, the separable exact solutions which then arise are a complete class of solutions, and there are no further non-separating solutions. Also, these solutions coincide with those in the WKB approximation. Thus in [4] 
we found solutions for which the WKB form is exact - this is a consequence of the chosen factor ordering. This implies that the lattice regularization is in fact equivalent to the WKB approximation, with the additional constraint that the wave functional of the cloud be a product of shell wave functionals.

In the present paper we used these exact solutions to derive Hawking radiation and the accompanying greybody factors for the non-marginal case, thus generalizing our earlier work

for the marginal case in [10]. Ideally, one would like to obtain quantum gravitational corrections to Hawking radiation - this, however, does not seem feasible within the framework of the presently used lattice regularization, since the WKB solution is also an exact solution, thereby ruling out any scope for a quantum gravity induced correction. (Interestingly, in 18] it has been suggested that such quantum gravitational corrections to the semiclassical Hawking radiation will be significant only above a certain minimum energy threshold).

Another very important application of a model of quantum gravitational collapse is to investigate whether or not the singularity predicted for classical collapse is avoided in the quantum theory. In the present context this would require us to construct wave packets out of the exact WKB solutions that we have constructed here. Unfortunately, that does not seem possible for the non-marginal case since the factor-ordering we are constrained to choose depends explicitly on the energy function $E$ (although one could perhaps construct wave packets with respect to $b)$. On the other hand, it may be possible to construct wave packets in the marginal case and we plan to investigate singularity avoidance in this case. Also, it would be of interest to investigate the LTB quantum collapse using the methods of loop quantum gravity, although in the loop approach one can at present strictly investigate only the cosmological singularity, and not the black hole singularity arising in an asymptotically flat spacetime.

It also seems useful at this stage to look beyond the lattice regularization, and investigate other regularization schemes. The Wheeler-DeWitt equation for the LTB model is analogous to a two-dimensional quantum field theory. Hence it will be worth trying to adopt regularization methods used for two-dimensional quantum field theories to the present gravitational context. We plan to take up such investigations for the quantum LTB model in the future.

\section{Acknowledgements}

T. P. S. gratefully acknowledges support from the German Science Foundation (DFG) under the grant 446 IND 113/34/0-1.

[1] C. Kiefer, Quantum Gravity. Second edition (Oxford University Press, Oxford, 2007).

[2] G. Lemaître, Annales de la Société Scientifique de Bruxelles A 53, 51 (1933); for an English translation, see Gen. Rel. Grav. 29, 641 (1997).

[3] A. Krasiński, Inhomogeneous Cosmological Models (Cambridge University Press, Cambridge, 1997). 
[4] C. Kiefer, J. Müller-Hill, and C. Vaz, Phys. Rev. D 73, 044025 (2006).

[5] K. V. Kuchař, Phys. Rev. D 50, 3961 (1994).

[6] C. Vaz and L. Witten, Phys. Rev. D 60, 024009 (1999).

[7] C. Vaz and L. Witten, Phys. Rev. D 63, 024008 (2001); ibid. 64, 084005 (2001).

[8] C. Vaz, L. Witten, and T. P. Singh, Phys. Rev. D 63, 104020 (2001).

[9] C. Vaz, L. Witten, and T. P. Singh, Phys. Rev. D 69, 104029 (2004).

[10] C. Vaz, C. Kiefer, T. P. Singh, and L. Witten, Phys. Rev. D 67, 024014 (2003).

[11] P. S. Joshi and T. P. Singh, Phys. Rev. D 51, 6778 (1995); Class. Quantum Grav. 13, 559 (1996).

[12] L. D. Landau and E. M. Lifshitz, The Classical Theory of fields. Fourth revised English edition (Butterworth Heinemann, 1999).

[13] K. Martel and E. Poisson, Am. J. Phys. 69, 476 (2001).

[14] S. W. Hawking, Commun. Math. Phys. 43, 199 (1975).

[15] N. D. Birrell and P. C. W. Davies, Quantum fields in curved space (Cambridge University Press, Cambridge, 1982).

[16] I. S. Gradshteyn and I. M. Ryzhik, Table of Integrals, Series, and Products, sixth edition (Academic Press, San Diego, 2000), p. 343.

[17] D. N. Page, Phys. Rev. D 13, 198 (1976); A. A. Starobinsky and S. M. Churilov, Sov. Phys. JETP 38, 1 (1974).

[18] I. Agullo, J. Navarro-Salas, G. J. Olmo, and L. Parker, hep-th/0611355. 